\title{
MEDIDAS DA CONDUTIVIDADE TÉRMICA EFETIVA DE MODELOS DE POLPAS DE FRUTAS NO ESTADO CONGELADO'
}

\author{
Jaime Vilela de RESENDE ${ }^{2, *}$, Vivaldo SILVEIRA JR ${ }^{3}$
}

\begin{abstract}
RESUMO
As propriedades termofísicas de alimentos, necessárias nas simulações e cálculos do processo de congelamento, incluem principalmente a densidade, condutividade térmica e calor específico. Neste trabalho, as difusividades e condutividades térmicas da solução, usadas como modelo para o congelamento de polpas de frutas, foram medidas pelo método da sonda com aquecimento. Os experimentos foram conduzidos na faixa de -25 a $0^{\circ} \mathrm{C}$ com modelos alimentícios constituídos de $0,5 \%$ de $\mathrm{K}$ - carrageenan $+10 \%$ de sacarose (massa/volume de água). Modelos estruturais foram usados para as avaliações da condução de calor, combinada com a fração de gelo predita para as amostras a partir dos modelos de Heldman e foram comparados com os valores das condutividades térmicas efetivas medidas. Os modelos estruturais empregados foram: em série, paralelo e Maxwell-Eucken, com o gelo considerado como a fase dispersa. Em todos os ensaios, o modelo de Maxwell-Eucken apresentou os melhores resultados (erro máximo de 6,13\% quando comparado com os valores experimentais medidos) e foi escolhido para a predição da condutividade térmica efetiva de soluções-modelo de polpas de frutas congeladas. Os valores calculados da condutividade térmica foram ajustados em termos de funções polinomiais, divididas em quatro faixas de temperatura e podem ser usadas na resolução dos problemas de transferência de calor, nos processos de congelamento. Palavras-chave: alimentos congelados; condutividade térmica; *polpa de fruta.
\end{abstract}

\section{SUMMARY}

MEASUREMENTS OF EFFECTIVE THERMAL CONDUCTIVITY OF FRUIT PULP MODELS IN THE FROZEN STATE. The thermo physical properties of foods required in the simulation of freezing processes include density, conductivity and specific heat. In this work, thermal diffusivities and thermal conductivities of fruit pulp models were measured using the hot wire probe method. Experiments were carried out from 0 to $-25^{\circ} \mathrm{C}$ with food models constituted by $0,5 \% \mathrm{~K}$-carrageenan $+10 \%$ sucrose (weight/volume in water). Structural models were used for evaluating heat conduction combined with ice fraction predicted from the Heldman models for the samples, and were compared with the measurement of effective thermal conductivity. The structural models used were: series, parallel and MaxwellEucken models with ice as the dispersed phase. With all the samples tested, the model, which was composed of the dispersed ice phase, showed the best results (underestimated on $6,13 \%$ when compared with experimental measurements) and was chosen for predicting the effective thermal conductivity of frozen fruit pulp model-solutions. The values calculated for the thermal conductivity were fitted in terms of polynomial functions of temperature, divided into four temperature ranges and could be used in the resolution of heat transfer problems in the freezing process.

Keywords: frozen foods; thermal conductivity; *fruit pulp.

\section{1 - INTRODUÇÃO}

O conhecimento das propriedades termofísicas de alimentos é necessário para o desenvolvimento de cálculos de transferência de calor que estão envolvidos nos projetos dos equipamentos de refrigeração e armazenamento de alimentos. Tais propriedades são essenciais para a simulação da variação da temperatura no interior dos alimentos durante o congelamento e são também importantes para as estimativas do tempo de congelamento e da carga térmica do produto.

A maioria dos alimentos têm um alto teor de umidade e conseqüentemente a água serve como um meio de dispersão dos constituintes da mistura alimentar. Assim, a queda do ponto de congelamento é observada em diversos sistemas alimentares. Entre 0 e $-40^{\circ} \mathrm{C}$ as propriedades termofísicas de alimentos mostram importantes mudanças, devido à variação contínua do conteúdo de gelo nesta faixa de temperatura [9].

Geralmente, nas determinações experimentais das propriedades térmicas de alimentos a maior dificuldade é atribuída à grande dependência destas proprie- dades e suas grandes variações em relação a baixas temperaturas características dos processos de congelamento.

Devido a várias complicações encontradas na avaliação experimental das propriedades térmicas durante o congelamento, os esforços estão concentrados em construir modelos matemáticos baseados nas propriedades termodinâmicas de uma solução ideal. A correlação matemática das propriedades térmicas de alimentos como uma função de sua composição química básica e conteúdo de água tem sido uma alternativa para a realização experimental [10]. Equações para a estimativa de propriedades termofísicas para sistemas com e sem mudanças de fase são facilmente encontradas na literatura $[1,2,3,4,5,7,11,12]$.

Grande parte dos estudos envolvendo o desenvolvimento de modelos matemáticos e medidas experimentais das propriedades térmicas de alimentos são realizados utilizando sistemas modelos e os resultados são aplicados para alimentos de composição similar. Para simular ensaios com polpas de frutas, tem-se usado soluções de sacarose e diferentes tipos de géis, com suas concen-

${ }^{1}$ Recebido para publicação em 07/08/01. Aceito para publicação em 04/01/02.

${ }^{2}$ LEST/FEMEC/UFU -Av. João Naves de Avila 2160 - Bloco 1 M-Campus Santa Mônica - CEP 38400-089 Uberlândia/MG juresende@mecânica.ufu.br

${ }^{3}$ DEA/FEA/UNICAMP - CX. Postal 6121 - Cid. Universitária Zeferino Vaz - CEP 13083-970 - Campinas/SP

* A quem a correspondência deve ser enviada. 
trações ajustadas de acordo com o teor de sólidos solúveis da polpa [3].

Os sistemas modelo são extremamente úteis quando se deseja estudar processos ou ainda verificar a exatidão de modelos matemáticos de predição. Substâncias escolhidas como modelo para a utilização em experimentos de congelamento devem ter suas propriedades termofísicas definidas e sua composição deve ser semelhante a do sistema alimentício real, tanto na quantidade quanto na natureza de seus constituintes.

\section{1 - Modelos estruturais para a condução de calor}

A condutividade térmica é uma propriedade não aditiva que não pode ser determinada somente a partir da composição de um material [8]. A estrutura do alimento afeta o tipo de formulação matemática para a avaliação da condutividade térmica efetiva. A relação entre a condutividade térmica intrínseca e a fração volumétrica de cada componente é descrita pela seguinte função:

$k_{e}=f\left(k_{1}, k_{2}, \ldots ; x_{1}^{v}, x_{2}^{v}, \ldots\right)$

onde $k_{1}, k_{2}, \ldots$ são os valores da condutividade térmica intrínseca para cada componente no modelo estrutural alimentar, e, $x_{1}{ }^{v}, x_{2}{ }^{v}, \ldots$ são as frações volumétricas de cada componente no sistema. Os modelos série, paralelo e Maxwell-Eucken (ME) ou disperso, são os mais freqüentemente aplicados na formulação destes modelos estruturais e são apresentados na Tabela 1 .

Neste trabalho, o objetivo foi determinar experimentalmente a condutividade e difusividade térmica em função da temperatura do sistema modelo, durante o processo de congelamento e compará-las com propriedades estimadas, usando modelos encontrados na literatura. Estes resultados podem ser utilizados como ferramentas na avaliação do fenômeno de transferência de calor durante o congelamento de polpas de frutas embaladas em sacos de polietileno e acondicionados em caixas.

TABELA 1. Modelos estruturais para a determinação da condutividade térmica de modelos alimentícios [8].



onde,

$$
x_{i}^{v}=\frac{\frac{x_{i}^{w}}{\rho_{i}}}{\sum \frac{x_{i}^{w}}{\rho_{i}}}
$$

Os subscritos se referem a $s=$ sólido; $w=$ água, $c=$ fase contínua, $d=$ fase dispersa, e os superescritos $v$ e $w$ se referem à fração volumétrica e a fração mássica, respectivamente.

\section{2 - MATERIAL E MÉTODOS}

\section{1 - Preparo das amostras}

O sistema modelo foi constituído de um gel composto de $0,5 \%$ (massa/volume de $\mathrm{H}_{2} \mathrm{O}$ ) de $\mathrm{K}$-carrageenan e $10 \%$ (massa/volume de $\mathrm{H}_{2} \mathrm{O}$ ) de sacarose. Para o preparo do gel, o pó de K-carrageenan, após a pesagem, foi adicionado à sacarose formando uma mistura sólida e homogênea dos dois ingredientes. À mistura foi em seguida adicionada lentamente uma pequena quantidade de água destilada fria, até a completa dispersão. A esta dispersão foi adicionada, sob agitação, água destilada em ebulição, em quantidade suficiente para se obter o gel na concentração desejada.

\section{2 - Montagem experimental}

Medidas da condutividade térmica " $k$ " e da difusividade " $\alpha$ " foram feitas, simultaneamente, usando o método da sonda [6] com aquecimento. A sonda foi construída com uma agulha hipodérmica de $0,6 \mathrm{~mm}$ de diâmetro externo e $100 \mathrm{~mm}$ de comprimento, no interior da qual foi colocada uma resistência de aquecimento (níquel-cromo) de $0,35 \mathrm{~m}$ de comprimento e $0,08 \mathrm{~mm}$ de diâmetro, juntamente com um pequeno termopar localizado exatamente no centro da sonda. Todos estes componentes foram cuidadosamente isolados com resina epoxi.

Uma potência de aquecimento linear de $17,5 \mathrm{~W} / \mathrm{m}$ foi aplicada à resistência por meio de uma fonte de corrente e o aumento da temperatura foi registrado através do sistema de aquisição de dados, a cada 2,0 segundos. Para cobrir a faixa de temperaturas especificada para o processo de congelamento, um banho criostático contendo solução de 50\% (volume/volume) de etanol em água foi utilizado e as temperaturas de estabilização das amostras foram acompanhadas com termômetros padrão (ASTM, USA). Os experimentos foram realizados em triplicata e a construção da sonda e montagem experimental podem ser visualizadas na Figura 1.

A difusividade é obtida pelo intercepto, B, e o gradiente $\mathrm{G}$ (coeficientes angulares), a partir da região de aumento na temperatura $\mathrm{T}(\mathrm{t})$ versus logaritmo do tempo t (equação 5). Para uma sonda com geração de calor por unidade de comprimento $\mathrm{q}_{\mathrm{l}}$, raio $\mathrm{R}$ e condutividade $\mathrm{k}_{\mathrm{p}}$ em um meio de condutividade $\mathrm{k}$ e difusividade $\alpha$, a equação para a elevação da temperatura $\mathrm{T}(\mathrm{t})$ a uma distância radial (r) torna-se, para um grande período de $\mathrm{t}$ [6]: 
$\mathrm{T}(\mathrm{t})=\mathrm{Gln} t+\mathrm{B}$

onde a partir do gradiente $\mathrm{G}=\mathrm{q}_{1} / 4 \pi \mathrm{k}$ e do intercepto $\mathrm{B}$, pode-se calcular $\mathrm{k}$ :

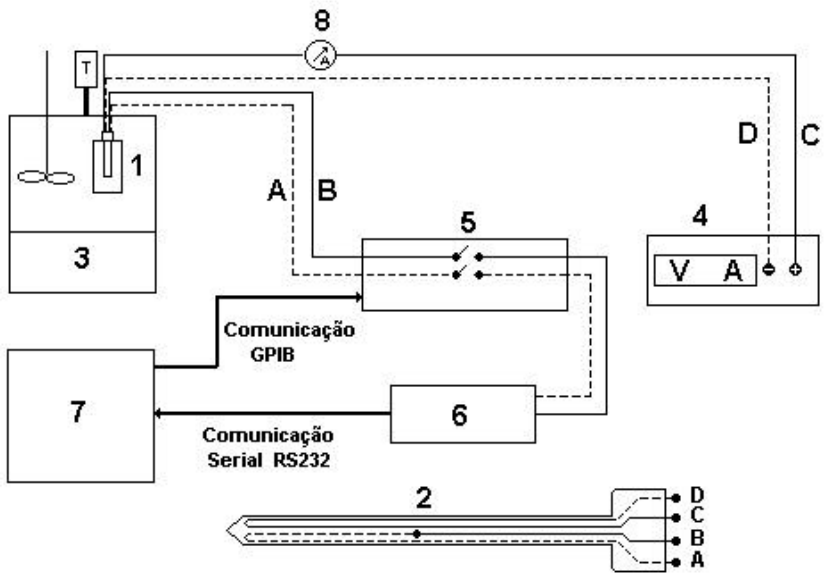

FIGURA 1. Montagem experimental da sonda com aquecimento para determinação da condutividade térmica dos sistemas modelo: 1) Amostra; 2) sonda com aquecimento; 3) ultracriostato; 4) Fonte de corrente; 5) scanner (chave seletora); 6) Conversor analógico/digital; 7) microcomputador; 8) amperimetro; A) fio constantan; B) fio cobre; C e D) alimentação da resistência.

$B=\frac{q_{1}}{4 \pi k}\left\{\ln \left(\frac{4 \alpha}{R^{2}}\right)-0,5772-\frac{2 k}{k_{p}} \ln \left(\frac{r}{R}\right)\right\}$

e após um rearranjo tem-se a difusividade $\alpha$ :

$\alpha=\frac{R^{2}}{4} \exp \left\{\frac{B}{G}+0,5772+\frac{q_{1}}{G} D\right\}$

e então $D=\frac{\ln (r / R)}{2 k_{p} \pi}$

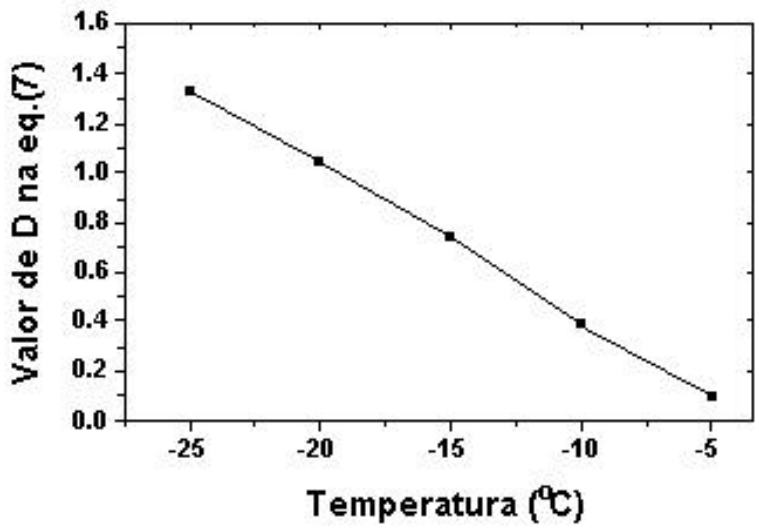

FIGURA 2. Valores de "D" obtidos para a calibração da sonda usando solução de $10 \%$ de sacarose adicionada de $4 \%$ de agar, de condutividade e difusividade térmicas conhecidas.
Os valores de D são característicos para a sonda e foram determinados pela calibração em materiais de difusividade térmica conhecida. Neste trabalho, a calibração da sonda foi feita com uma solução de sacarose $10 \%$ com adição de $4 \%$ (massa/volume de $\mathrm{H}_{2} \mathrm{O}$ ) de agar, sendo que os valores da condutividade e difusividade térmicas utilizados, foram os encontrados na literatura [9].

O gráfico apresentado na Figura 2 mostra os valores de D obtidos utilizando solução de sacarose 10\% e agar $4 \%$ para estabelecer uma potência de aquecimento de $17,5 \mathrm{~W} / \mathrm{m}$ para a sonda nestas condições.

\section{3 - Predição da condutividade térmica efetiva a partir do ponto de congelamento inicial do modelo}

As estimativas dos coeficientes de condutividade térmica efetiva para a solução modelo de sacarose $(10 \%)$ e K-carrageenan $(0,5 \%)$ foram feitas através dos modelos descritos pelas equações (2), (3) e (4), dos modelos série, paralelo e Maxwell-Eucken, respectivamente.

Para este último, existe a necessidade de se estimar precisamente a fração de gelo do modelo, que varia com a temperatura de congelamento. Para esta finalidade, uma vez que se conhece o ponto de congelamento inicial da solução, a partir dos valores médios provenientes das curvas de resfriamento da solução modelo, faz-se uso das equações descritas por HELDMAN [5], para formular a queda do ponto de congelamento da solução modelo e predizer a relação entre a fração de água não congelada e a temperatura durante o congelamento. Para a solução modelo de sacarose (15\%) adicionada de $\mathrm{K}$-carrageenan $(0,5 \%)$, a temperatura de início do congelamento obtida dos históricos de temperaturas é $\mathrm{T}_{\text {ic }}=-0,73^{\circ} \mathrm{C}$ ou $272,42 \mathrm{~K}$.

Os valores da condutividade térmica intrínseca para a sacarose, determinada a partir da condutividade térmica efetiva no estado congelado pela aplicação destes três métodos, foram obtidos de MIYAWAKI, PONGSAWATMANIT [8] e estão apresentados na Tabela 2.

TABELA 2. Condutividade térmica intrínseca da sacarose como componente de solutos em modelos alimentares [8].

\begin{tabular}{cccc}
\hline Modelo & Série & Paralelo & ME \\
\hline $\mathrm{k}_{\text {saca }}(\mathrm{W} / \mathrm{mK})$ & 0,345 & 0,257 & 0,293 \\
\hline
\end{tabular}

No estado congelado, um sistema é composto de três fases: a fase gelo, a fase água não congelada e a fase do soluto. As propriedades térmicas destes materiais puros, necessários para calcular a condutividade térmica efetiva usando estes modelos, estão listadas na Tabela 3 $[8,9]$.

Para o modelo Maxwell-Eucken, foi considerado que o gelo está disperso em uma segunda fase composta de água não congelada e sólidos. A condutividade térmica desta segunda fase foi calculada aplicando novamente o modelo disperso, considerando, nesta etapa, os sóli- 
dos como meio contínuo e a água não congelada como a fase dispersa.

TABELA 3. Condutividade térmica e densidade de componentes puros

\begin{tabular}{ccc}
\hline & Material & Equação $\left[\mathbf{k}(\mathrm{W} / \mathrm{mK})=\mathbf{f}\left(\mathbf{T}\left({ }^{\circ} \mathrm{C}\right)\right)\right]$. \\
\hline Cond. Intrínseca & Água & $0,5711+1,763 \times 10^{-3} \mathrm{~T}-6,704 \times 10^{-6} \mathrm{~T}^{2}$ \\
$k$ & Gelo & $2,220-6,249 \times 10^{-3} \mathrm{~T}+1,015 \times 10^{-4} \mathrm{~T}^{2}$ \\
$(\mathrm{~W} / \mathrm{m} \mathrm{K})$ & Sacarose & $0,304+9,93 \times 10^{-4} \mathrm{~T}$ \\
\hline Densidade & Água & $997,2+3,144 \times 10^{-3} \mathrm{~T}$ \\
$\rho$ & Gelo & $916,9-0,1307 \mathrm{~T}$ \\
$\left(\mathrm{~kg} / \mathrm{m}^{3}\right)$ & Sacarose & $1599-0,3105 \mathrm{~T}$ \\
\hline
\end{tabular}

\section{3 - RESULTADOS E DISCUSSÃO}

\section{1 - Condutividade e difusividade térmica}

A Figura 3 apresenta os resultados experimentais da resposta da variação da temperatura em função do tempo, produzida pela sonda de aquecimento registrada pela aquisição de dados, para a temperatura de $-20,4^{\circ} \mathrm{C}$, após a estabilização das amostras no banho criostático. Também é apresentado na Figura 3 o aumento da temperatura $\mathrm{T}(\mathrm{t})$ versus o logaritmo do tempo, para a obtenção dos parâmetros G e B, das equações 5 e 6 . A Figura 4 apresenta a parte linear da curva $\mathrm{T}=\mathrm{f}(\ln \mathrm{t})$, e os valores da inclinação $\mathrm{G}$ e do intercepto $\mathrm{B}$ usados para a determinação da condutividade térmica e da difusividade a partir das equações 5 e 6 , bem como o grau de correlação da linearidade nesta parte da curva experimental, para as respectivas temperaturas.

A Figura 5 mostra a média dos resultados dos dados experimentais obtidos pelo método da sonda com aquecimento, para a solução modelo de K-carrageenan $(0,5 \%)$, adicionada de sacarose (10\%), utilizando as equações 5 a 8. Observa-se no gráfico, uma importante variação nas propriedades térmicas a baixas temperaturas, devido à alta variação da fração de gelo nesta faixa. Segundo RENAULD et al. [9], na faixa entre -40 a $-10^{\circ} \mathrm{C}$ a concentração de sólidos é o principal fator, influenciando a quantidade de gelo formado a uma determinada temperatura, sendo que esta quantidade diminui à medida que aumenta a concentração de sólidos.

A comparação da predição da condutividade térmica da solução modelo obtida a partir dos três modelos (equações 2 a 4) na faixa de temperatura compreendida entre -30 e $0^{\circ} \mathrm{C}$ comparada com os resultados obtidos a partir dos dados experimentais também é apresentada na $\mathrm{Fi}$ gura 5.

Para o cálculo da condutividade térmica efetiva da solução modelo, os valores da condutividade térmica intrínseca dos solutos utilizados foram aqueles apresentados na Tabela 2. Nota-se que a condutividade térmica intrínseca varia de modelo para modelo. MIYAWAKI, PONGSAWATMANIT [8] justificam o fato da dependência do valor intrínseco em relação ao modelo estrutural inerente. $\mathrm{O}$ modelo em série fornece resistências mais altas à condução de calor, o modelo paralelo resistências menores e o modelo Maxwell- Eucken é intermediário.
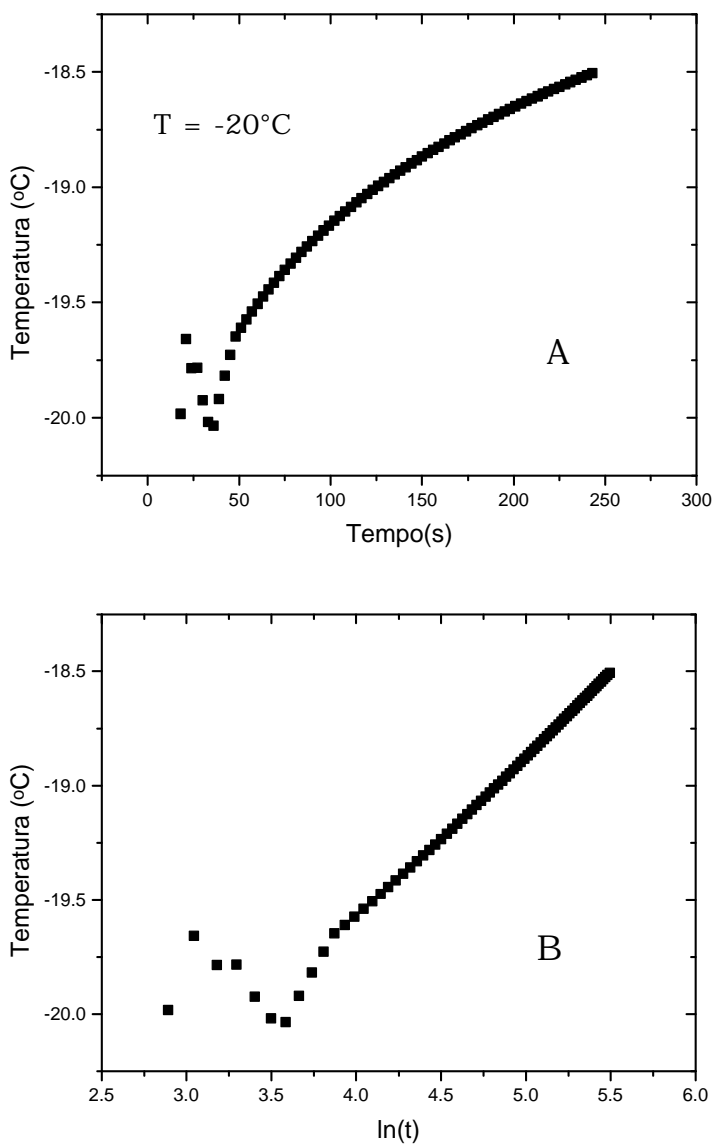

FIGURA 3. (A) Resultados experimentais da variação da temperatura em função do tempo, produzida pela sonda de aquecimento. (B) Gráfico do aumento da temperatura $\mathrm{T}(\mathrm{t})$ versus $\ln (\mathrm{t})$. Condições experimentais: Potência de aquecimento $(\mathrm{P}=$ $17,5 \mathrm{~W} / \mathrm{m})$; Temperatura inicial das amostras $\left(\mathrm{T}_{\mathrm{b}}=-20,4^{\circ} \mathrm{C}\right)$.

A Tabela 4 mostra uma comparação da condutividade térmica medida experimentalmente e os valores obtidos pelos três modelos. O erro percentual é calculado a partir da seguinte equação:

$$
\% \text { Erro }=\frac{k_{\text {exp }}-k_{p r e d}}{k_{\exp }} \times 100 \%
$$

Como se pode observar, o modelo Maxwell-Eucken, obteve a melhor predição da condutividade térmica da solução modelo, quando comparado aos outros dois modelos (série e paralelo). O modelo Maxwell-Eucken tem sido aplicado freqüentemente para a avaliação da condutividade térmica de modelos alimentícios.

Para o cálculo dos modelos preditivos o alto peso molecular atribuído a K-carrageenan foi considerado no cálculo do peso molecular efetivo dos sólidos, mas não foi incluído como um componente que pudesse causar diferenças significativas da condutividade térmica da solução modelo. Sua condutividade intrínseca não foi 
considerada nos cálculos do modelo, sendo esta atribuída apenas à sacarose. Este procedimento pode ser justificado, analisando os dados obtidos por KENT et al. [6] para a condutividade térmica de um modelo não conge- lado formado por um gel constituído de $98 \%$ de água e $2 \%$ de K-carrageenan comparando estes dados com aqueles obtidos para água pura nas mesmas condições, como mostrado na Tabela 5.



FIGURA 4. Parte linear da curva $\mathrm{T}=\mathrm{f}(\ln t)$ para obtenção dos valores da inclinação G e do intercepto $\mathrm{B}$ usados nas equações (5) a (8). 


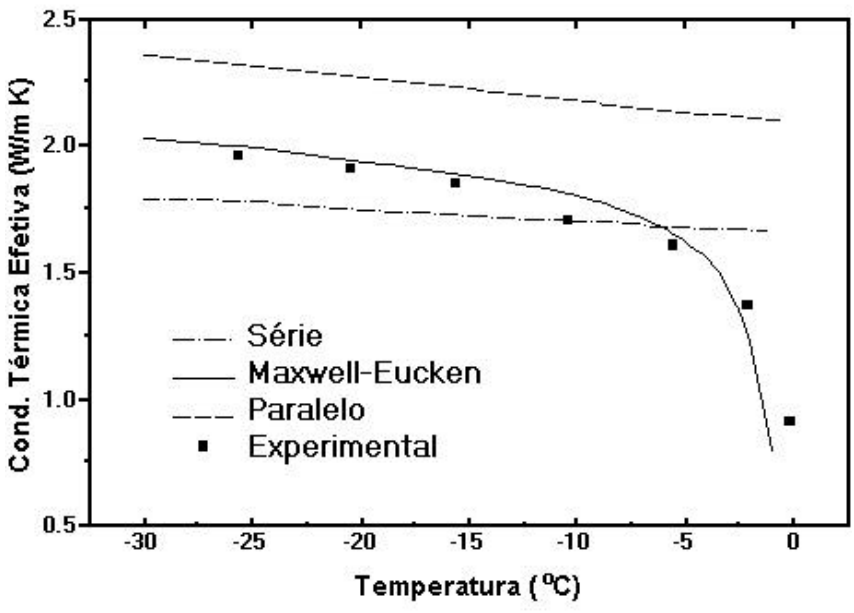

FIGURA 5. Condutividades térmicas efetivas para a solução de sacarose (10\%) e K-carrageenan $(0,5 \%)$, nas temperaturas de congelamento preditas pelos modelos estruturais em série, paralelo e Maxwell-Eucken, em comparação com os resultados experimentais obtidos.

TABELA 4. Comparação entre os resultados experimental e predito para a condutividade térmica efetiva da solução modelo.

\begin{tabular}{|c|c|c|c|c|c|c|c|}
\hline \multirow[b]{2}{*}{$\begin{array}{c}\mathrm{T} \\
\left({ }^{\circ} \mathrm{C}\right)\end{array}$} & \multirow[b]{2}{*}{$\mathrm{k}_{\text {Exp }}$} & \multirow[b]{2}{*}{$\begin{array}{c}\text { kSérie } \\
(\mathrm{W} / \mathrm{m} \mathrm{K})\end{array}$} & \multirow[b]{2}{*}{ \%Erro } & \multirow[b]{2}{*}{$\begin{array}{l}\text { KParalelo } \\
(\mathrm{W} / \mathrm{m} \mathrm{K})\end{array}$} & \multicolumn{3}{|c|}{$\mathrm{k}_{\text {Maxwell- }}$} \\
\hline & & & & & \%Erro & $\begin{array}{l}\text { Eucken } \\
(\mathrm{W} / \mathrm{m} \mathrm{K})\end{array}$ & \%Erro \\
\hline$-5,6$ & 1,605 & 1,6734 & $-4,26$ & 2,1320 & $-32,8$ & 1,6623 & $-3,57$ \\
\hline$-10,4$ & 1,70 & 1,6928 & 0,42 & 2,1675 & $-27,5$ & 1,8043 & $-6,13$ \\
\hline$-15,6$ & 1,85 & 1,7164 & 7,22 & 2,2108 & $-19,5$ & 1,8819 & $-1,72$ \\
\hline$-20,4$ & 1,91 & 1,7402 & 8,89 & 2,255 & $-18,1$ & 1,9360 & $-1,361$ \\
\hline$-25,6$ & 1,96 & 1,7683 & 9,78 & 2,3086 & $-17,79$ & 1,9891 & $-1,49$ \\
\hline
\end{tabular}

Observa-se que existe uma pequena diferença entre os valores da condutividade térmica do gel quando se utiliza uma concentração de $2 \%$ quando comparados ao da água pura. No presente trabalho, a concentração de $\mathrm{K}$-carrageenan é cerca de quatro vezes inferior àquela apresentada na Tabela 5, podendo-se então esperar que a participação da condutividade intrínseca deste componente no modelo estrutural seja menor.

TABELA 5. Comparação entre as condutividades térmicas intrinsecas para um gel formado por $98 \%$ de água e $2 \%$ de $\mathrm{k}$ carrageenan e para a água pura, nas mesmas condições [6].

\begin{tabular}{ccccc}
\hline $\begin{array}{c}\text { Temperatura } \\
\left({ }^{\circ} \mathrm{C}\right)\end{array}$ & $\begin{array}{c}\mathrm{k}_{\text {gel }}(98 \% \text { água }) \\
\mathrm{W} / \mathrm{m} \mathrm{K}\end{array}$ & $\begin{array}{c}\text { Desvio } \\
\text { Padrão }\end{array}$ & $\begin{array}{c}\text { kágua } \\
\mathrm{W} / \mathrm{m} \mathrm{K}\end{array}$ & $\begin{array}{c}\text { Diferença } \\
(\%)\end{array}$ \\
\hline 10 & 0,592 & 0,031 & 0,579 & $+2,19$ \\
25 & 0,609 & 0,018 & 0,606 & $+0,49$ \\
40 & 0,645 & 0,022 & 0,627 & $+2,79$ \\
\hline
\end{tabular}

A Tabela 6 mostra os resultados obtidos para as difusividades térmicas obtidas a partir da curva de aumento de temperatura da sonda pela resolução da equação (7).
TABELA 6. Difusividades térmicas obtidas a partir da curva de aquecimento da sonda, utilizando a equação (7).

\begin{tabular}{cc}
\hline Temperatura $\left({ }^{\circ} \mathrm{C}\right)$ & Difusividade Térmica \\
\hline$-5,6$ & $2,50 \times 10^{-7} \mathrm{~m} \cdot \mathrm{s}^{-2}$ \\
$-10,4$ & $1,33 \times 10^{-7} \mathrm{~m} \cdot \mathrm{s}^{-2}$ \\
$-15,6$ & $6,15 \times 10^{-7} \mathrm{~m} \cdot \mathrm{s}^{-2}$ \\
$-20,4$ & $7,01 \times 10^{-7} \mathrm{~m} \cdot \mathrm{s}^{-2}$ \\
$-25,6$ & $7,55 \times 10^{-7} \mathrm{~m} \cdot \mathrm{s}^{-2}$ \\
\hline
\end{tabular}

\section{2 - Condutividade térmica efetiva em função da temperatura de resfriamento}

Os dados resultantes das equações de predição, apresentados na Figura 5, foram submetidos a uma rotina de regressão polinomial para a obtenção de funções polinomiais dependentes da temperatura, para a faixa de temperatura de -35 a $0^{\circ} \mathrm{C}$. Os valores dos coeficientes usados para aproximar por mínimos quadrados, os valores calculados da condutividade térmica em termos do grau de funções polinomiais da temperatura de congelamento, para a solução modelo de K-carrageenan (0,5\% massa/volume de água) e sacarose (10\% massa) volume de água), específicas para este trabalho são apresentadas na Tabela 7.

TABELA 7. Coeficientes das funções polinomiais para o cálculo da condutividade térmica efetiva da solução modelo, em função da temperatura $\left({ }^{\circ} \mathrm{C}\right)$ de resfriamento.

\begin{tabular}{|c|c|c|c|c|c|c|c|c|c|}
\hline \multicolumn{10}{|c|}{ Equação Polinomial: $y=a+b T+c T^{2}+d T^{3}+\ldots$} \\
\hline Faixa de $\mathrm{T}^{\circ} \mathrm{C}$ & Prop & $a$ & $\mathrm{~b}$ & $\mathrm{c}$ & $d$ & $\mathrm{e}$ & f & g & $\mathrm{R}^{2}$ \\
\hline$-1,5<\mathrm{T} \leq \mathrm{T}_{\mathrm{ic}}$ & $\mathbf{k}$ & $-0,76159$ & $-2,61797$ & $-1,30562$ & $-0,26224$ & & & & 1 \\
\hline$-6,0<T \leq-1,5$ & $\mathbf{k}$ & $-0,52808$ & $-2,15482$ & $-1,09727$ & $-0,33744$ & $-0,06089$ & $-0,00592$ & $-2,389 \mathrm{E}-4$ & 1 \\
\hline$-14,0<T \leq-6,0$ & k & 0,96116 & $-0,28436$ & $-0,04003$ & $-0,00322$ & $-1,364 \mathrm{E}-4$ & $-2,374 \mathrm{E}-6$ & & 1 \\
\hline$T \leq-14,0$ & $\mathbf{k}$ & 1,54574 & $-0,05645$ & $-0,00292$ & $-9,623 \mathrm{E}-4$ & $-1,622 \mathrm{E}-6$ & $-1,118 \mathrm{E}-8$ & & 1 \\
\hline
\end{tabular}

${ }^{*} \mathrm{k}=\mathrm{k}(\mathrm{T})$ é a Condutividade Térmica $\left(\mathrm{W} / \mathrm{m}{ }^{\circ} \mathrm{C}\right)$;

\section{4 - CONCLUSÕES}

Os valores da condutividade térmica da solução modelo foram obtidos e analisados matematicamente, na faixa de temperatura de interesse. Como resultado, o modelo de Maxwell-Eucken, considerando o gelo puro como a fase dispersa, apresentou-se melhor entre todos os casos testados, sugerindo a sua aplicabilidade para a predição da condutividade térmica da solução modelo durante o congelamento, podendo suprir de forma satisfatória a carência de dados na literatura para o sistema modelo em questão. Os valores obtidos do modelo de Maxwell-Eucken subestimaram os valores experimentais com um erro máximo em torno de $-6,2 \%$ enquanto nos demais modelos estes foram superiores, levando a subestimativas de até $-33 \%$, no caso do modelo paralelo.

As equações apresentadas na Tabela 7 permitem 0 cálculo da condutividade térmica efetiva do produto (solução modelo) diretamente, sendo necessário apenas o conhecimento dos históricos das temperaturas obtidos durante o processamento, disponibilizando dados indis- 
pensáveis aos cálculos de transferência de calor e análise final do processo de congelamento.

\section{5 - REFERÊNCIAS BIBLIOGRÁFICAS}

[1] BECKER, B. R.; FRICKE, B. R. Food Thermophysical Property Models. International Communication on Heat and Mass Transfer, v. 26, n. 5, p. 627-636, 1999.

[2] CLELAND, A. C. Food Refrigeration Process. Analysis, Design and Simulation. Elsevier Applied Science: London e New York. 1992. 284p.

[3] DA SILVA, S. B. Propriedades Termofísicas de Polpa de Abacaxi. Campinas, 1994. 94p. Tese de Mestrado, Faculdade de Engenharia de Alimentos, Universidade Estadual de Campinas (UNICAMP).

[4] FIKIIN,K.A.; FIKIIN, A. G. Predictive equations for thermophysicals properties and enthalpy during cooling and freezing of food materials. Journal of Food Engineering, v. 40, p. 1-6, 1999.

[5] HELDMAN, D. R. Food Freezing. In: HELDMAN, D. R.; LUND, D. B. (eds.) Handbook of Food Engineering, New York; Marcel Dekker, 1992. 756p.

[6] KENT, M.; CHRISTANSEN, K.; van HANEGHEM,I.A.; HOLTZ, E.; MORLEY, M. J.; NESVBA, P.; POULSEN, K. P. Cost 90 Collaborative measurements of thermal properties of foods. Journal of Food Engineering, v. 3, p. 117-150, 1984.

[7] MANNAPERUMA, J. D.; SINGH, R. P. A Computer-Aided Method for the Prediction of Properties and Freezing/ Thawing Times of Foods. Journal of Food Engineering, v. 9, p. 275-304, 1989.

[8] MIYAWAKI, O.; PONGSAWATMANIT, R. Mathematical analysis of the effective thermal conductivity of food materials in the frozen state. Bioscience, Biotechnology Biochemistry, v. 58, n. 7, p. 1222-1225, 1994.

[9] RENAUD, T.; BRIERY, P.; ANDRIEU, J.; LAURENT, M. Thermal Properties of food materials in the frozen state, Journal of Food Engineering, v. 15, p. 83-97, 1992.

[10] SAAD, Z.; SCOTT, E. P. Estimation of temperature dependent thermal properties of basic food solutions during freezing. Journal of Food Engineering, v. 28, p. 1-19, 1996.

[11] SUCCAR, J. Estimation of thermophysical properties of food at freezing temperatures. ASHRAE Transactions, part 2B, p. 312-332, 1985.

[12] SUCCAR, J.; HAYAKAWA, K. Empirical Formulae for Predicting Thermal Physical Properties of Food at Freezing or Defrosting Temperatures. Lebensmittel Wissenchaft und Technolology., v. 16, p. 326-331, 1983. 